\title{
Associated production with onia, double onia production at the LHC
}

\author{
Evelina Bouhova-Thacker ${ }^{* \dagger}$ \\ Lancaster University \\ E-mail: e.bouhovadcern.ch
}

The most recent results on associated production with onia and double onia production at the LHC are presented and compared to theoretical predictions and measurements by other experiments.

The 15th International Conference on Flavor Physics and CP Violation

5- 9 June, 2017

Prague, Czech Republic

\footnotetext{
* Speaker.

${ }^{\dagger}$ On behalf of the ATLAS, CMS and LHCb Collaborations.
} 


\section{Introduction}

Much progress has been made in understanding heavy flavour production at the LHC since the start of data taking and the results by the ATLAS [1], CMS [2] and LHCb [3] Collaborations presented here can help further disentangle the contributions from different production mechanisms. Quarkonia (onia) are formed from a heavy quark and its antiquark ( $c \bar{c}$ or $b \bar{b}$ ). Double onia and associated onia production can distinguish between the production of a heavy quark system in colour-singlet (CS) and colour-octet (CO) states and determine the fraction of events produced through double parton scattering (DPS), which is important for the understanding of background in many searches for new physics phenomena.

Two objects can be produced in the same $p p$ collision either in a single interaction of two partons or through the simultaneous interaction of two pairs of partons, each producing one of the two objects. The inverse coefficient of proportionality between the cross section for the production of two objects and the product of cross sections for the production of each object is called the effective cross section:

$$
\sigma_{A+B}^{\mathrm{DPS}}=\frac{1}{1+\delta_{A B}} \frac{\sigma_{A} \sigma_{B}}{\sigma_{\mathrm{eff}}} \quad \delta_{A B}=\left\{\begin{array}{ll}
1, & A=B \\
0, & A \neq B
\end{array} .\right.
$$

$\sigma_{\text {eff }}$ is expected to be largely independent of the scattering process and centre-of-mass energy, indeed all existing measurements are between 2 and $20 \mathrm{mb}$.

Double parton scattering is not distinguishable from single parton scattering (SPS) on an eventby-event basis but they are expected to differ in overall kinematic features, such as angular correlations, for example the difference in the rapidities or the azimuthal angles of the two objects. Large uncertainties come from possible higher-order SPS contributions, feed-down and limited knowledge of the transverse profile of the proton.

\section{Associated production with onia}

ATLAS has published results on the associated production of $J / \psi$ mesons and $W$ bosons $[\llbracket$ at centre-of-mass energy of $\sqrt{s}=7 \mathrm{TeV}$, and $J / \psi$ mesons and $Z$ bosons [5] at $\sqrt{s}=8 \mathrm{TeV}$.

The separation of prompt and non-prompt signal and backgrounds in associated $J / \psi$ and $W$ production is done using an unbinned maximum likelihood (ML) fit to the $J / \psi$ invariant mass and its pseudo-proper time. Non-prompt $J / \psi$ mesons are produced in decays of $b$-hadrons and can mimic DPS. The signal yield is extracted in a fit to the weighted $W$ boson transverse mass using templates. The DPS yield is obtained using a value of $\sigma_{\text {eff }}=15 \pm 3(\text { stat })_{-3}^{+5}$ (syst) $\mathrm{mb}$, taken from the $W^{ \pm} \rightarrow \ell v_{\ell}+2-$ jet measurement [6].

The signal yields are used to determine the ratio of the $W+$ prompt $J / \psi$ cross sections to the inclusive $W^{ \pm}$production cross section. After corrections for the fiducial acceptance of the muons from $J / \psi$, the inclusive and the DPS subtracted ratios of cross sections are obtained and compared to LO CS and NLO CO predictions (Figure 1). The presence of both SPS and DPS contributions is observed, with SPS being the dominant production mechanism, especially at low $J / \psi$ transverse momenta as can be seen from the inclusive differential cross section distribution, Figure 1 (b). 
Figure 11(c) shows the difference in azimuthal angles between $J / \psi$ and W. It is expected to be flat for DPS and peaking at $\pi$ (rad) for SPS.

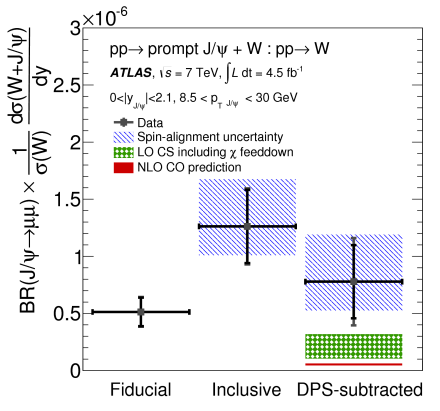

(a)

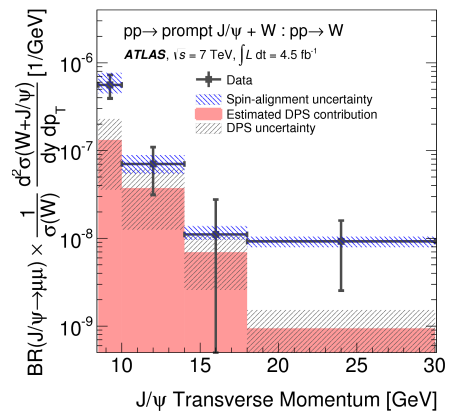

(b)

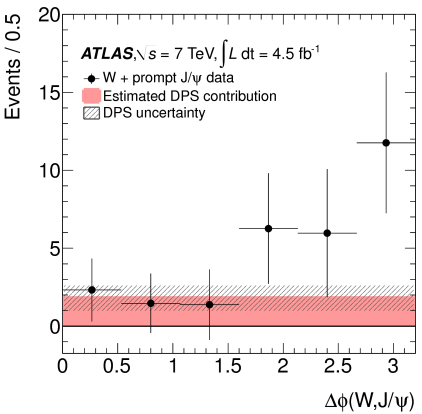

(c)

Figure 1: (a) The $W^{ \pm}+$prompt $J / \psi: W$ production cross section ratio in the $J / \psi$ fiducial region (Fiducial), after correction for $J / \psi$ acceptance (Inclusive), and after subtraction of the double parton scattering component (DPS-subtracted). (b) The inclusive (SPS+DPS) cross section ratio $d R_{J / \psi}^{\text {incl }} / d p_{T}$ as a function of $J / \psi$ transverse momentum. (c) Azimuthal angle between the $W^{ \pm}$and $J / \psi$ for prompt $J / \psi$ candidates; all plots taken from [ [ $]$.

The strategy for the ATLAS measurement of prompt and non-prompt $J / \psi$ production in association with a $Z$ boson is similar to the $J / \psi+W$ analysis, but with a template fit to the $Z$ invariant mass. Total and DPS signal yields are reported for prompt and non-prompt $J / \psi$ separately. Assuming that all observed signal in the first bin of the differential distribution for prompt events, as a function of $\Delta \phi$ in Figure $2(a)$, is due to DPS, a lower limit is set on the effective cross section of $5.3 \mathrm{mb}$.

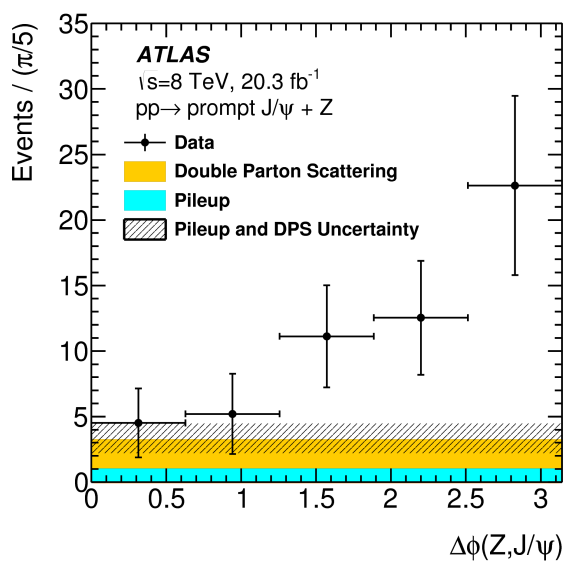

(a)

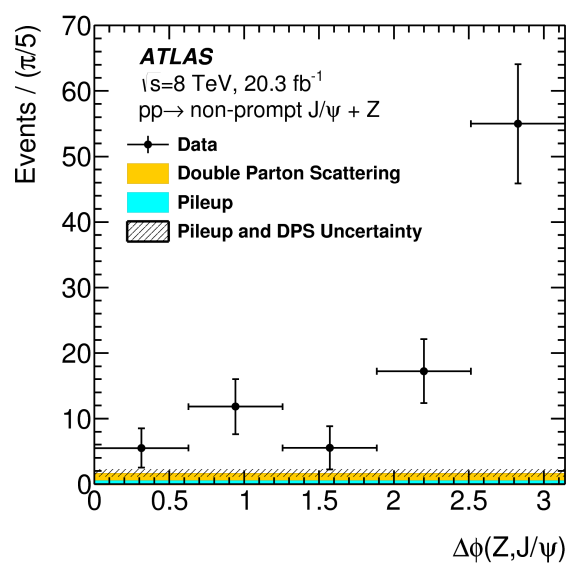

(b)

Figure 2: Azimuthal angle between the $Z$ boson and the $J / \psi$ meson for (a) prompt and (b) non-prompt signal; plots taken from [5].

The ratios of the $Z+J / \psi$ cross sections and the DPS fraction to the inclusive $Z$ cross section are obtained and a comparison of the DPS subtracted ratio to NLO CS and CO models and a LO CS model are shown in the Figure 3 (a). The expected production rate for the sum of NLO CO 
and CS is lower than the data by a factor of 2 to 5 with the discrepancy increasing with transverse momentum as can be seen from the inclusive differential cross section ratio shown in Figure 3 (b).

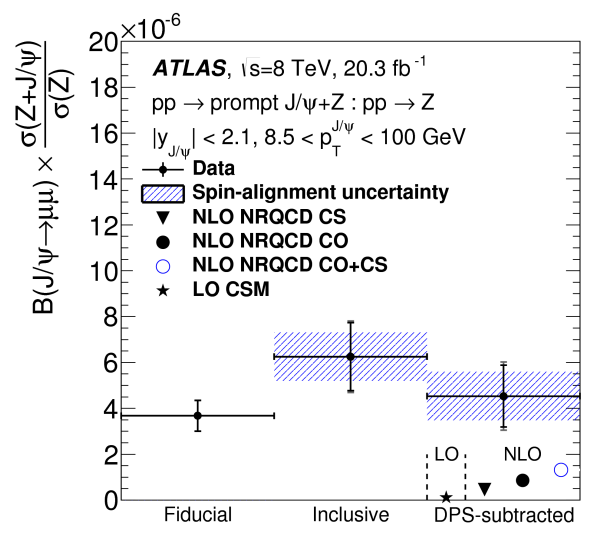

(a)

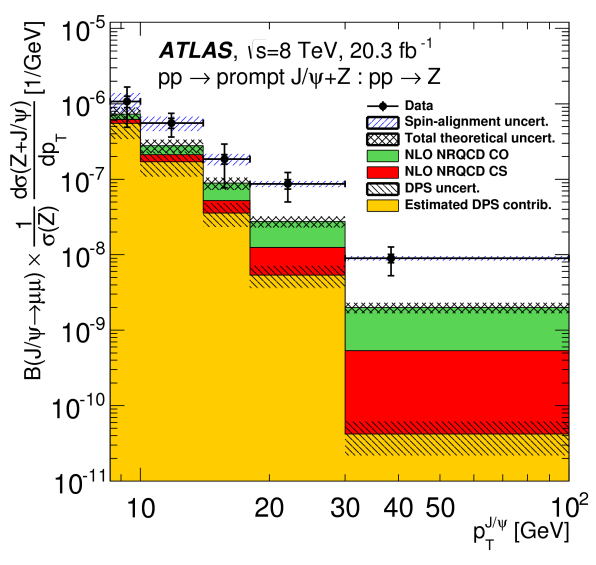

(b)

Figure 3: (a) The $Z+$ prompt $J / \psi: Z$ production cross section ratio in the $J / \psi$ fiducial region (Fiducial), after correction for $J / \psi$ acceptance (Inclusive), and after subtraction of the double parton scattering component (DPS-subtracted). (b) $Z+$ prompt $J / \psi$ production cross section ratio as a function of $J / \psi$ transverse momentum; plots taken from [5].

The LHCb has performed measurements of associated production of $J / \psi[7]$ and $\Upsilon$ [8] with open charm states $(C)$. The open charm states considered and their decay modes are: $D^{0}\left(\rightarrow K^{-} \pi^{+}\right)$, $D^{+}\left(\rightarrow K^{-} \pi^{+} \pi^{+}\right), D_{s}^{+}\left(\rightarrow\left(K^{+} K^{-}\right)_{\phi} \pi^{+}\right)$and $\Lambda_{c}^{+}\left(\rightarrow p K^{-} \pi^{+}\right)$. The $J / \psi+C$ analysis is performed on the $7 \mathrm{TeV}$ data, the $\Upsilon+C$ on 7 and $8 \mathrm{TeV}$ data. The signal yields are obtained from an unbinned ML fit to the 2D onia and charm mass distributions.

The model independent production cross sections in the fiducial volume, after correcting for acceptance, trigger, reconstruction, selection and particle identification efficiencies, are obtained for the $\Upsilon+D^{0}$ and $\Upsilon+D^{+}$states, separately for the two centre-of-mass energies. The ratios of these final states to the inclusive $\Upsilon(1 S)$ production and the ratio of $\Upsilon(1 S)+c \bar{c}$ to the inclusive $\Upsilon(1 S)$ production are in good agreement with the DPS prediction and significantly larger than the SPS prediction. The measurements for the $J / \psi+C$ cross sections in the 4 channels are compared to LO SPS predictions and the DPS estimate. Here too the SPS predictions are significantly smaller than observed and a better agreement with DPS is seen.

Neglecting the SPS contributions allows an estimate for the effective cross section to be made: $\sigma_{\text {eff }}^{\Upsilon(1 S) D^{0,+}}=18.0 \pm 1.8 \mathrm{mb}$, which is in good agreement with multi-jet production measurements at the Tevatron and the LHC.

The two LHCb papers provide a number of differential measurements for the cross sections of onia + charm. The $\Delta \phi$ and $\Delta y$ distributions included in Figure $\emptyset$ show a good agreement with the DPS expectations as the main production mechanism for associated onia and charm.

\section{Double onia production}

Double onia production has been studied by CMS [9], ATLAS [10] and LHCb [11] in the case of $J / \psi$ pair production and by CMS [12] in the case of $\Upsilon(1 S)$ pair production. The signal yields 

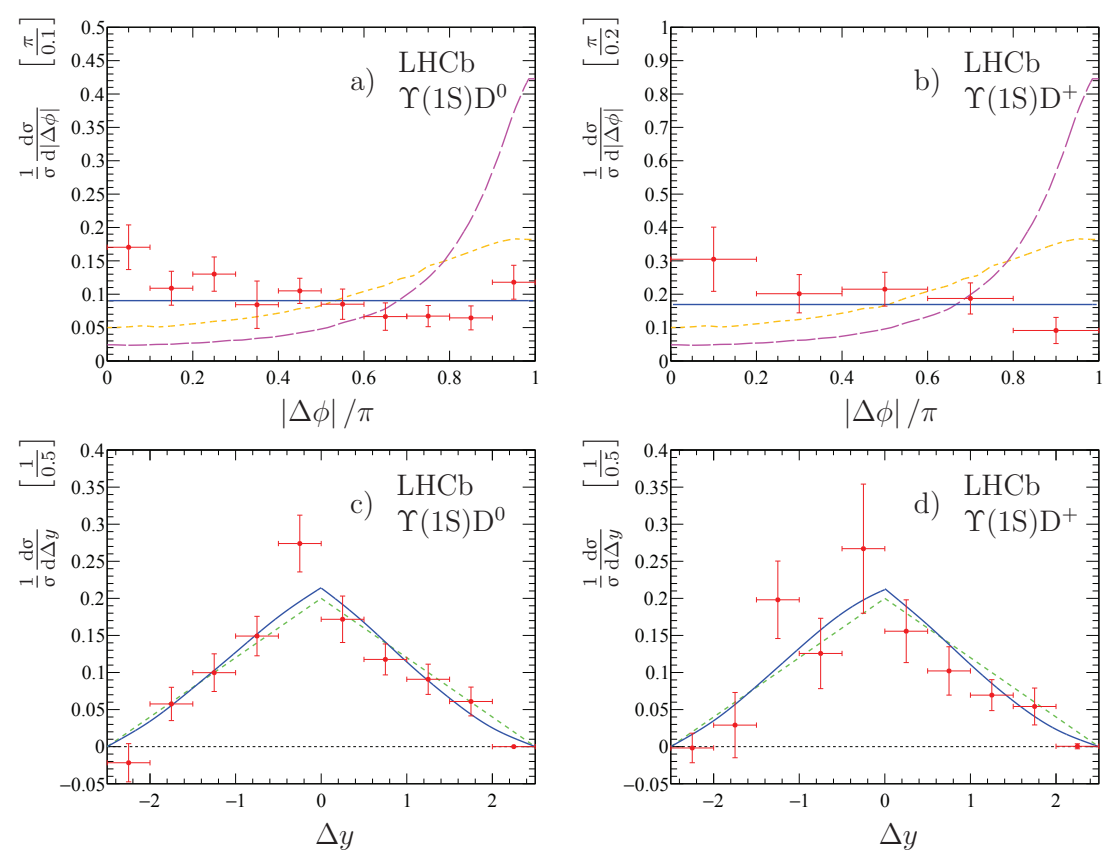

Figure 4: Background-subtracted and efficiency-corrected distributions for $|\Delta \phi| / \pi$ (top) and $\Delta y$ (bottom) for $\Upsilon(1 S) D^{0}$ (left) and $\Upsilon(1 S) D^{+}$(right) events; plots taken from [8].

are extracted from unbinned extended ML fits to the invariant mass distributions of the two onia. The early Run $1 \mathrm{CMS}$ result using $7 \mathrm{TeV}$ data reports the cross section for $J / \psi$ pair production. The differential cross section as a function of the rapidity difference between the two $J / \psi$ (Figure 5] is sensitive to DPS and was used by Lansberg and Shao [13] to extract a value for the effective cross section: $\sigma_{\text {eff }}=8.2 \pm 2.2 \mathrm{mb}$, assuming the last bin is populated entirely by DPS.

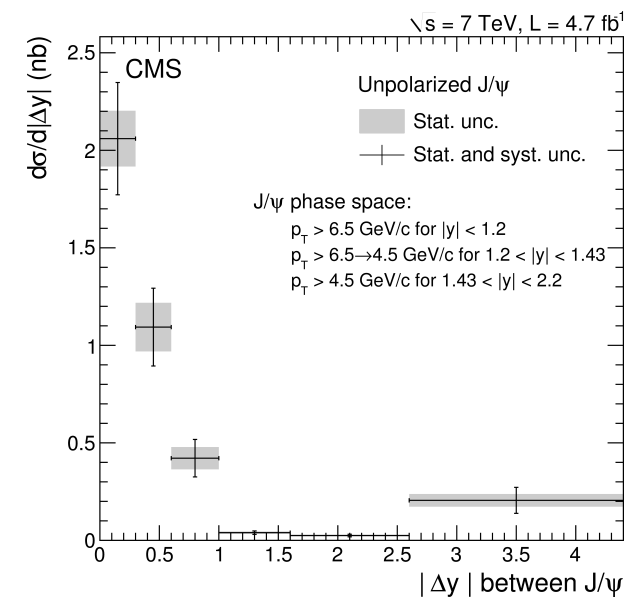

Figure 5: Differential cross section for prompt $J / \psi$ pair production as a function of the absolute rapidity difference between the $J / \psi$ mesons; plot taken from [p].

The ATLAS prompt-prompt di- $J / \psi$ signal is extracted using the $8 \mathrm{TeV}$ data and the fiducial and inclusive cross sections are reported for 2 rapidity regions $(|y|<1.05$ and $1.05<|y|<2.1)$ based 
on the sub-leading $J / \psi$ rapidity. The fraction of DPS events is determined by fitting DPS and SPS templates to the data in bins of $\Delta y$ and $\Delta \phi$. The resulting DPS cross section is used to extract $\sigma_{\text {eff }}^{J / \psi J / \psi}=6.3 \pm 1.6$ (stat) \pm 1.0 (syst) $\pm 0.1(\mathrm{BF}) \pm 0.1$ (lumi) $\mathrm{mb}$. The value is consistent with the existing results for $\sigma_{\text {eff }}$ from di- $J / \psi$ measurements, which are lower than the values obtained from other final states.

The total and DPS cross sections in the full rapidity range in the muon fiducial volume are compared to NLO* SPS and LO DPS predictions (Figure 6). The data is largely in agreement with the sum of NLO* SPS and LO DPS predictions. Contributions from feed-down and intrinsic parton transverse momentum may account for the remaining discrepancies.

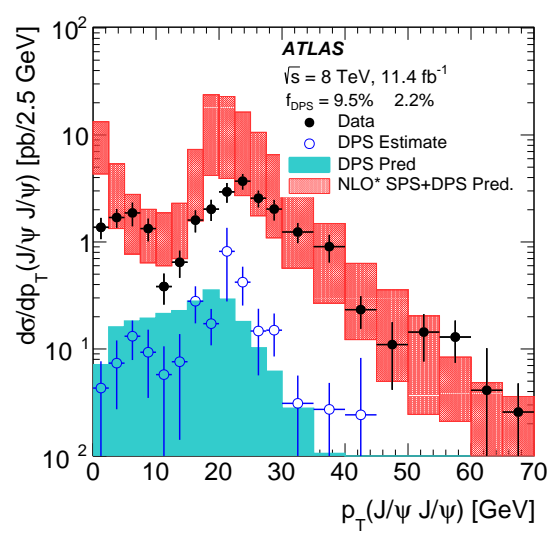

(a)

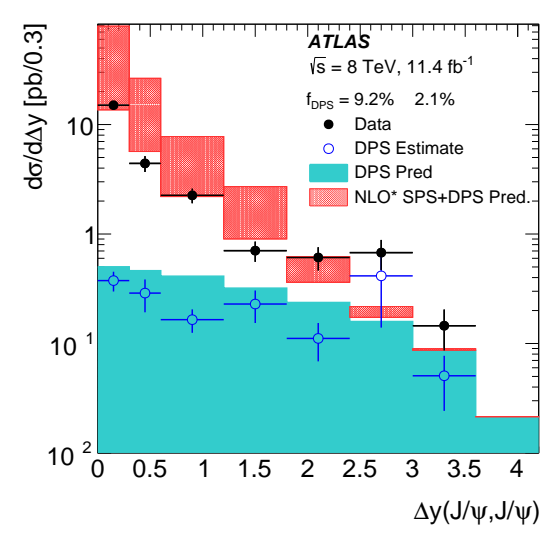

(b)

Figure 6: The DPS and total differential cross sections as a function of the (a) transverse momentum of the di- $J / \psi$ and (b) difference in rapidity between the two $J / \psi$ mesons; plots taken from [10].

The LHCb $J / \psi$ pair production result uses Run 2 data at $\sqrt{s}=13 \mathrm{TeV}$. The signal yield and di- $J / \psi$ production cross section are determined using an unbinned ML fit to the $2 \mathrm{D}$ mass distributions and include a fraction of non-prompt $J / \psi$. The cross section is compared to a number of theoretical predictions, which vary considerably, with the best agreement provided by NLO* CS models. Assuming all $J / \psi$ pairs are produced by DPS, an estimate of the effective cross section is made: $\sigma_{\text {eff }}^{J / \psi J}=7.3 \pm 0.5$ (stat) \pm 1.0 (syst) $\mathrm{mb}$.

The differential production cross sections as a function of several kinematic variables, some of which are shown in Figure 7, are compared to the predictions of the different theoretical models considered. Neither the DPS model, nor any of the SPS models can describe simultaneously the measured cross section and the differential shapes, but a sum can. A simple template fit to the differential distributions for the different SPS models is used to extract the DPS fraction. All fits indicate a large DPS contribution with the CO contribution being significantly smaller than the CS one. The DPS fraction values translate into corresponding values for $\sigma_{\text {eff }}$, which vary between 10 and $12.5 \mathrm{mb}$. These values are smaller than the LHCb measurement from associated onia production but slightly larger than the central production measurements by ATLAS and CMS and the Tevatron for double $J / \psi$ production.

For the CMS measurement of $\Upsilon(1 S)$ pair production at $8 \mathrm{TeV}$, signal yields are obtained considering di- $\Upsilon(1 S)$ and $\Upsilon(2 S)+\Upsilon(1 S)$ production. The invariant mass distributions of the two $\Upsilon$ 


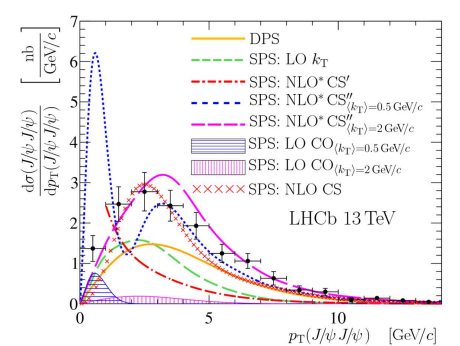

(a)

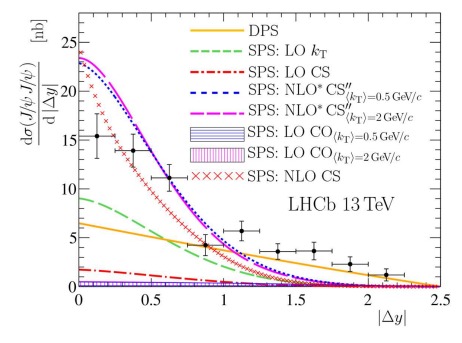

(b)

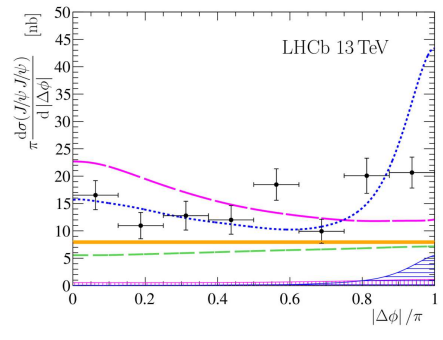

(c)

Figure 7: Comparisons between measurements and theoretical predictions for the differential cross sections as a function of (a) $p_{T}(J / \psi J / \psi)$, (b) $\Delta y$ and (c) $\Delta \phi$; plots taken from [11].

candidates are shown in Figure 8 .
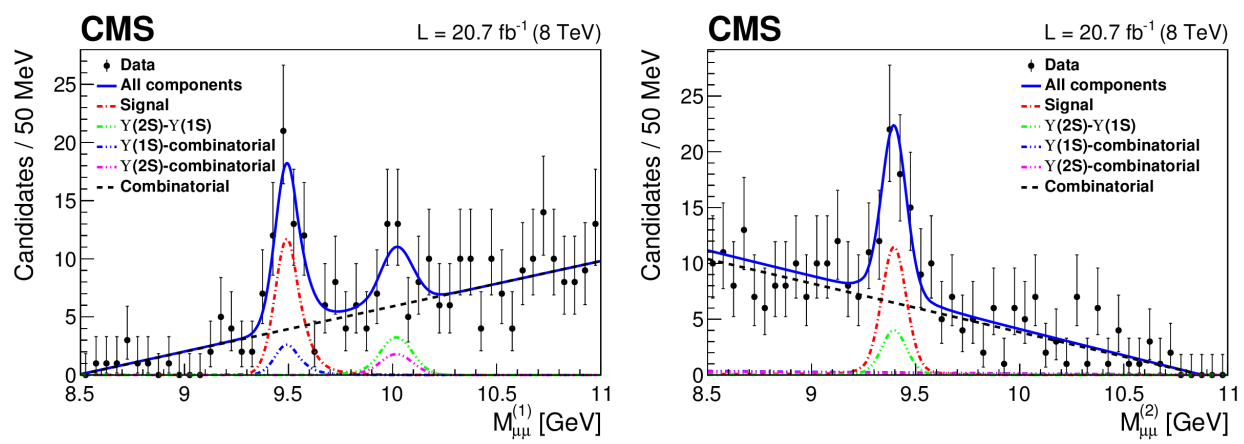

Figure 8: Invariant mass distributions of the higher-mass muon pair (left) and the lower-mass muon pair (right); plots taken from [12].

The cross section for di- $\Upsilon(1 S)$ is measured and assuming a conservatively low value for the fraction of DPS of $10 \%$ results in a $\sigma_{\text {eff }}$ value of $6.6 \mathrm{mb}$. The DPS fraction estimated from a prediction [14] for the the cross section for $\Upsilon(1 S)$ pair production, which assumes SPS with feeddown from higher $\Upsilon$ states, yields a value of $30 \%$, corresponding to $\sigma_{\text {eff }}$ of $2.2 \mathrm{mb}$. The range 2.2 to $6.6 \mathrm{mb}$ is smaller than the values obtained in multi-jet measurements and similar to the ones from central di- $J / \psi$ production.

\section{Conclusions}

Several results from the LHC experiments shedding light on double and associated onia production have been published so far. The data is largely in agreement with the NLO* SPS + LO DPS predictions, although there are indications that contributions from feed-down and the effects of the intrinsic parton transverse momenta should be included to explain all aspects of the observed distributions.

Apart from the $13 \mathrm{TeV} \mathrm{LHCb}$ result in the forward rapidity region, most double onia results from ATLAS, CMS and the Tevatron, are generally lower than the values obtained from other final states, such as different multi-jet states as well as the associated production with onia studied by ATLAS and LHCb. 


\section{References}

[1] ATLAS Collaboration, The ATLAS Experiment at the CERN Large Hadron Collider, JINST 3 (2008) S08003.

[2] CMS Collaboration, The CMS experiment at the CERN LHC, JINST 3 (2008) S08004.

[3] LHCb Collaboration, The LHCb Detector at the LHC, JINST 3 (2008) S08005.

[4] ATLAS Collaboration, Measurement of the production cross section of prompt $J / \psi$ mesons in association with a $W^{ \pm}$boson in pp collisions at $\sqrt{s}=7$ TeV with the ATLAS detector, JHEP 04 (2014) 172.

[5] ATLAS Collaboration, Observation and measurements of the production of prompt and non-prompt $J / \psi$ mesons in association with a $Z$ boson in pp collisions at $\sqrt{s}=8 \mathrm{TeV}$ with the ATLAS detector, Eur. Phys. J. C75 (2015) 229.

[6] ATLAS Collaboration, Measurement of hard double-parton interactions in $W(\rightarrow \ell v)+2$ jet events at $\sqrt{s}=7$ TeV with the ATLAS detector, New J. Phys. 15 (2013) 033038.

[7] LHCb Collaboration, Observation of double charm production involving open charm in pp collisions at $\sqrt{s}=7 \mathrm{TeV}, \mathrm{JHEP} 06$ (2012) 141 .

[8] LHCb Collaboration, Production of associated $\Upsilon$ and open charm hadrons in pp collisions at $\sqrt{s}=7$ and 8 TeV via double parton scattering, JHEP 07 (2016) 052.

[9] CMS Collaboration, Measurement of prompt $\mathrm{J} / \psi$ pair production in pp collisions at $\sqrt{\mathrm{s}}=7 \mathrm{TeV}$, JHEP 09 (2014) 094.

[10] ATLAS Collaboration, Measurement of the prompt $J / \psi$ pair production cross-section in pp collisions at $\sqrt{s}=8 \mathrm{TeV}$ with the ATLAS detector, Eur. Phys. J. C77 (2017) 76.

[11] LHCb Collaboration, Measurement of the $J / \psi$ pair production cross-section in pp collisions at $\sqrt{s}=$ 13 TeV, JHEP 06 (2017) 047.

[12] CMS Collaboration, Observation of $\Upsilon(1 S)$ pair production in proton-proton collisions at $\sqrt{s}=8 \mathrm{TeV}$, JHEP 05 (2017) 013.

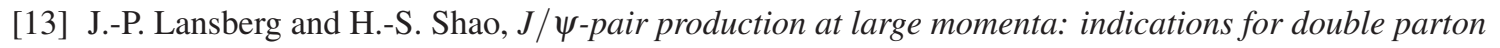
scatterings and large $\alpha_{s}^{5}$ contributions, Phys. Lett. B751 (2015) 479.

[14] A. V. Berezhnoy, A. K. Likhoded and A. A. Novoselov, ؟-meson pair production at LHC, Phys. Rev. D 87 (2013) 054023. 